\title{
RESEÑAS
}

De Prácticas y discursos Universidad Nacional del Nordeste Centro de Estudios Sociales I Año 8, Número 11, 2019, Marzo I ISSN 2250-6942

\section{EL MAPA Y LOS TERRITORIOS DE LOS ESTUDIOS DE GUBERNAMENTALIDAD. RESEÑA DEL LIBRO CONDUCTAS QUE IMPORTAN. VARIANTES DE ANÁLISIS DE LOS ESTUDIOS EN GUBERNAMENTALIDAD}

Emiliano Venier ${ }^{1}$

1 Docente e investigador de la Universidad Nacional de Salta. Correo electrónico: emilianovenier@yahoo.com.ar
Los Estudios de Gubernamentalidad (EG) constituyen una de las derivas intelectuales incitadas por las reflexiones realizadas por Michel Foucault durante la segunda mitad de la década de 1970, a partir de la recuperación de la expresión "gubernamentalidad" que permitió revisar su teoría sobre las relaciones de poder y construir una noción que permita pensar las racionalidades y las condiciones de emergencia de las técnicas y procedimientos para dirigir el comportamiento humano. En estas cuatro décadas, desde ese gesto fundacional del pensador francés, la difusión y recepción de las indagaciones inscriptas en estas preocupaciones han producido en el campo de las ciencias sociales, las humanidades y particularmente en la teoría política, un fecundo espacio en el que confluyen los esfuerzos para responder a las inquietudes por la construcción de los objetos de gobierno y el modo en que se plantea gobernarlos. Para el sociólogo alemán Thomas Lemke, desde este enfoque se posibilitó la apertura a diversas problematizaciones que permitieron la formulación de hipótesis de trabajo sobre la constitución reciproca de técnicas de poder y formas de conocimiento, y de regimenes de representación y modos de intervención, pero también para configurar las múltiples y diversas relaciones entre la institucionalización de organismos estatales y las formas históricas de subjetivación. Con la intencionalidad de abordar el derrotero que han tenido aquellas incitaciones foucaultianas y las singulares apropiaciones que en distintos puntos del planeta tuvieron eco para contribuir con la construcción de una analítica del presente, Aldo Avellaneda y Guillermo Vega compilan una serie de artículos de investigadores con antecedentes de peso en el campo de los Estudios de Gubernamentalidad. El título pensado, Conductas que importan. Variantes de análisis de los Estudios en Gubernamentalidad, ya resulta una provocación a su lectura, instalando sin rodeos la cuestión de las conductas como el núcleo de las pretensiones gubernamentales en las sociedades occidentales contemporáneas en las que las formas de subjetividad que definen las experiencias -o el límite de las experiencias- y los vínculos con uno mismo están marcados por racionalidades interesadas por la orientación de las conductas y de las existencias. Desde el 
De Prácticas y discursos

Universidad Nacional del Nordeste

Centro de Estudios Sociales

Año 8, Número 11, 2019, Octubre

ISSN 2250-6942

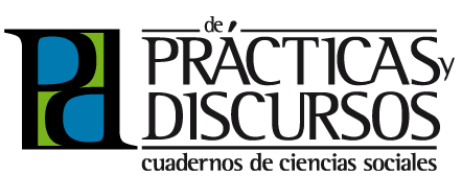

vamos hay una especie de empellón que puede des-ubicar al lector poco prevenido de las preocupaciones nodales de Foucault, provocando momentáneamente un distanciamiento de los valores que alientan las racionalidades neoliberales (si es que pudiera afirmarse la existencia de tal cosa) como los de autosuperación, la autorregulación y la continua capitalización del individuo. Uno encuentra en esta apuesta editorial el abordaje de un conjunto de planteamientos teóricos y metodológicos que explora más allá de los lugares comunes habilitados por las reflexiones derivadas del pensamiento foucaultiano. En este sentido, el libro aporta en la parte introductoria y a lo largo de sus capítulos un diagnóstico que permite, por un lado, la captura de ciertos modos en que se ensamblan las artes de gobierno para organizar, orientar y/o prescribir los comportamientos de los individuos. Por otro lado, se complementa con el mapa y la narrativa de las derivas de la tradición de los EG, cuyo trazado contribuye a reconocer las apuestas y los compromisos intelectuales, al tiempo que permite comprender en toda su dimensión epistemológica la propuesta analítica.

Algo que se señala como punto de partida en Conductas que importan... es la heterogeneidad, diversidad y dinámica de las prácticas de gobierno neoliberales que tienen su punto de apoyo en el estímulo creciente de libertades para producir márgenes de acción a elección de los individuos, pero que en su ejercicio se muestra cada vez más ajustado por técnicas de gobierno con herramientas legales y normativas. Pero los autores dejan entrever que las técnicas neoliberales no se remiten solamente a producir y regular el juego de las libertades, sino que resulta necesario no desdeñar en el análisis el funcionamiento del Estado, los poderes globales o extraterritoriales y las técnicas menos "soft" para dar cuenta del ímpetu de las diversas formas que el neoliberalismo propone como ejercicio del poder. En ese registro, sin renunciar al abandono de lo que Foucault denominara como "la hipótesis represiva" del poder, parece importante relevar y analizar las formas en que las racionalidades de gobierno apelan a prácticas donde la violencia aparece como elemento productivo de comportamiento.

Estos planteos aparecen fundamentados en la introducción elaborada por los compiladores y en el primer capítulo, denominado "Territorios observados", que cuenta con artículos de Thomas Lemke ("Foucault, política y error. Una revisión crítica de los estudios de gubernamentalidad"), Victoria Haidar ("¿Autoritarismo al 
De Prácticas y discursos

Universidad Nacional del Nordeste

Centro de Estudios Sociales

Año 8, Número 11, 2019, 0ctubre

ISSN 2250-6942
El mapa y los territorios de los estudios de gubernamentalidad. Reseña del libro conductas que importan. Variantes de análisis de los estudios en gubernamentalidad

interior del liberalismo? Entre los aportes de los Governmentality Studies y las posibilidades abiertas por la historia del Presente"), Ana Grondona ("Lost in translation? Una mirada periférica sobre los Governamentality Studies") y Stuart Elden ("Gubernamentalidad, cálculo, territorio").

En el libro también es posible encontrar toda una reflexividad sobre las herramientas metodológicas y conceptuales que permitirá a los interesados en esta línea de análisis apuntalar la grilla y ajustar el rigor en la labor investigativa -reconociendo sus posibilidades y sus limites-, actitud que, lejos de buscar el purismo o plantearse una postura academicista, debe comprenderse como una apuesta por inyectarle una mayor potencia al ejercicio de la crítica. En este esfuerzo por revisar las categorias con las que se opera en los Estudios de Gubernamentalidad se revisan los gestos señalados por Foucault y trabajados por los teóricos de esta tradición, tales como las ideas de problematización en relación con el gobierno, la relevancia analítica de las prácticas de gobierno; la grilla analítica, lo multiescalar y territorial en el análisis; la incapacidad explicativa de los "universales políticos"; la sustancialización del poder, la impugnación a la idea monolítica del Estado en las racionalidades políticas; las dificultades de disociar Estado, mercado y sociedad; o reconocer el papel plural y heterogéneo de los procesos de control, así como el rol productivo de los saberes y las racionalidades; entre otros. En este aspecto el libro, además, provoca el deseo de volver a aquellos pasajes de textos reconocidos como fundacionales de los Estudios de Gubernamentalidad y de aquellos que dieron entidad e institucionalidad a esta tradición.

Los artículos que componen Conductas que importan... se encuentran organizados en tres capitulos a partir de los cuales se va configurando la densidad de la trama de los EG: territorios observados, territorios expandidos, territorios operativos. En el primer capítulo, junto con la introducción de Guillermo Vega, Aldo Avellaneda, los textos de Thomas Lemke ("Foucault, política y error. Una revisión crítica de los estudios de gubernamentalidad"), Victoria Haidar ("¿Autoritarismo al interior del liberalismo? Entre los aportes de los Governmentality Studies y las posibilidades abiertas por la historia del Presente"), Ana Grondona ("Lost in translation? Una mirada periférica sobre los Governamentality Studies") y Stuart Elden ("Gubernamentalidad, cálculo, territorio") aportan, desde distintos ámbitos geográficos, esa reflexión 
De Prácticas y discursos

Universidad Nacional del Nordeste

Centro de Estudios Sociales

Año 8, Número 11, 2019, Octubre

ISSN 2250-6942

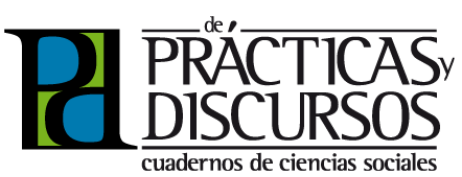

crítica mencionada más arriba en torno a las posibilidades y limitaciones o deficiencias de la grilla analítica de los EG.

En el segundo capítulo se encuentran los trabajos de Aldo Avellaneda ("El pensamiento, el afuera, el contexto. Las artes de gobierno y la historia del pensamiento político"), Carlos Bacchi ("¿Por qué estudiar las problematizaciones? Haciendo visible la política") y Guillermo Vega ("Gubernamentalidad jurídica, o de cómo podría abordarse la -problemática- presencia de la ley en la reflexión sobre la práctica de gobierno"), los cuales dan cuenta del movimiento expansivo de los dominios y objetos de los EG en el campo de las ciencias sociales.

En el tercer capítulo, los escritos no recalan tanto en la ponderación de los EG y su potencia explicativa, sino que abordan casos a partir de algún elemento del instrumental o de tematizaciones próximas. Con esta pretensión, los artículos de Pat O'Malley ("'La incertidumbre nos hace libres'. Riesgo, seguro y libertad"), Luciano Nosetto ("Gubernamentalidad, democracia y justicia. Una evaluación de la perspectiva gubernamental en vista de la judicialización de la política argentina"), Daniel Chao ("Problematización, gobierno y veteranos de la guerra de Malvinas") y Alejandro Ruidrejo ("Las misiones jesuíticas del Paraguay: neoliberalismo y poder pastoral") permiten hacerse de una idea más cabal de los abordajes que son admitidos en el territorio.

Conductas que importan. Variantes de análisis de los Estudios en Gubernamentalidad resulta una obra cuya contribución se puede medir por las reflexiones que cada uno de los artículos propone, pero también por una apuesta por afirmar la institucionalidad de un espacio de labor académica que asume la dispersión de un archipiélago que encuentra vinculaciones en las pretensiones de encontrar los elementos significativos para repensar los modos en que se despliegan las técnicas de gobierno y todo aquello que se articula en el complejo proceso de producir sujetos de gobierno: los juegos de verdad, las técnicas de poder y las formas de subjetividad que definen la experiencia de los individuos en el presente. 